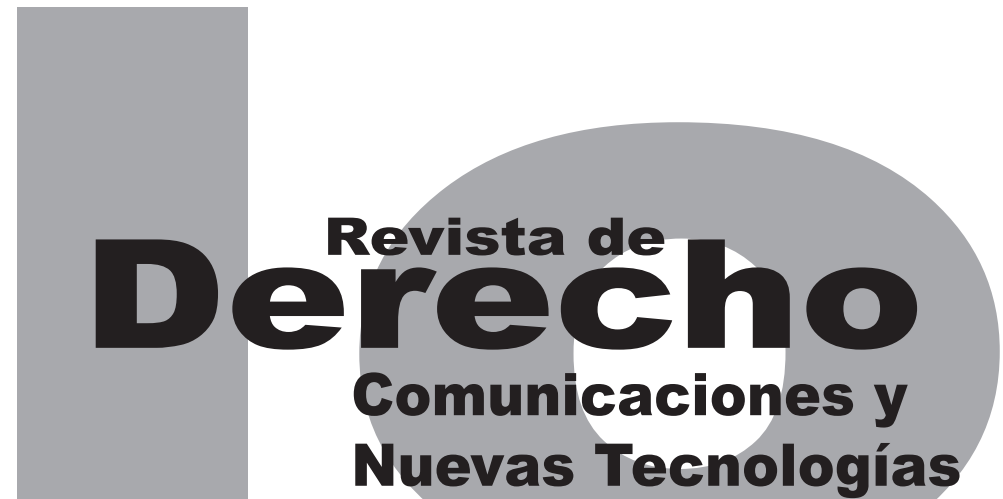

\title{
RESPONSABILIDAD INTERNACIONAL POR DAÑOS CAUSADOS POR OBJETOS ESPACIALES
}

\author{
Gladys Andrea Contreras Pasuy
}

Artículo de reflexión

Universidad de los Andes

Facultad de Derecho

Revista de Derecho, Comunicaciones y Nuevas Tecnologías

No. 11, Enero - Junio de 2014. ISSN 1909-7786 


\section{Responsabilidad internacional por daños causados por objetos espaciales}

\section{Resumen}

Desde su origen, la carrera espacial ha sido una actividad única por los avances tecnológicos que ha traído consigo. Sin embargo, también ha representado un riesgo mayor para la humanidad y el medioambiente. Esto ha hecho necesaria la creación de una regulación internacional especial que, por regla general, responsabilice de manera absoluta a los Estados que realicen o promuevan actividades espaciales desde su territorio o instalaciones. Esta tipología de responsabilidad impide que los Estados se exoneren de reparar los daños causados, aunque aleguen haber obrado de manera diligente. Este artículo indaga sobre la filosofía garantista que motiva esta regulación, que propende por que las víctimas reciban una reparación eficiente.

Palabras clave: Derecho Espacial, Derecho Internacional, responsabilidad, objeto espacial, daño, Estado de registro, Estado de lanzamiento.

\section{International liability for damages caused by space objects}

\section{Abstract}

Since its beginnings, the space race has been a unique activity because of the technological advances that has brought. However, it has also increased risks for humanity and the environment. This made necessary the creation of a special international regulation. By general rule, this regulation is considered liable in all means by the States that practice or promote space activities from their own territory or installations. This typology of liability impedes that States exonerate themselves from repairing damages caused by them, even if they allege their own diligence. This article inquires on the guarantee philosophy behind this special regulation, which promotes that victims receive reparation of caused damages efficiently.

Keywords: International Space Law, International Law, liability, space object, damage, State of registry, launching State.

\section{Responsabilidade internacional por danos causados por objetos espaciais}

\section{Resumen}

Desde sua origem, a corrida espacial tem sido uma atividade única pelos avanços tecnológicos que tem trazido consigo. Porém, também tem representado um risco maior para a humanidade e o meio ambiente. Isto tem tornado necessária a criação de uma regulação internacional especial que, por regra geral, responsabilize de maneira absoluta os Estados que realizem ou promovam atividades espaciais desde seu território ou instalações. Esta tipologia de responsabilidade impede que os Estados se exonerem de reparar os danos causados, embora aleguem ter obrado de maneira diligente. Este artigo indaga sobre a filosofia garantista que motiva esta regulação, que propende por que as vítimas recebam uma reparação eficiente.

Palavras-chave: Direito espacial, direito internacional, responsabilidade, objeto espacial, dano, Estado de registro, Estado de lançamento. 


\section{SUMARIO}

Introducción - I. ANTECEDENTES HISTÓRICOS Y NORMATIVOS DE LA CARRERA ESPACIAL - II. CONCEPTOS RELEVANTES EN MATERIA DE RESPONSABILIDAD INTERNACIONAL POR DAÑOS CAUSADOS POR OBJETOS ESPACIALES - III. RESPONSABILIDAD CONJUNTA EN EL DERECHO ESPACIAL - IV. TIPOLOGÍA DE RESPONSABILIDAD APLICABLE - V. PROCEDIMIENTO DE RECLAMACIÓN - VI. FILOSOFÍA TRAS LA TIPOLOGÍA DE RESPONSABILIDAD APLICABLE AL DERECHO ESPACIAL - VII. CONCLUSIONES - Referencias 


\title{
Responsabilidad internacional por daños causados por objetos espaciales ${ }^{1}$
}

\author{
Gladys Andrea Contreras Pasuy²
}

Introducción

En los últimos sesenta años, la carrera espacial desarrollada por los humanos ha determinado muchas de las circunstancias de la vida actual. Esto se ve plasmado, principalmente, en el ámbito de las telecomunicaciones y del conocimiento científico. De ahí que muchos concuerden en que "es difícil no considerar los viajes al espacio ineludibles" (Lachs, 1977, p. 15). En este sentido, desde su génesis en el siglo XX, la carrera espacial ha sido continua, y se prevé que continúe sin cesar.

Una de las principales acciones emprendidas por el ser humano en la carrera espacial ha sido la de "poblar" el espacio ultraterrestre con satélites artificiales. Estos satélites son objetos espaciales que, en su dinámica habitual, cum-

Cómo citar este artículo: Contreras Pasuy, G. A. (Junio, 2014). Responsabilidad internacional por daños causados por objetos espaciales. Revista de Derecho, Comunicaciones y Nuevas Tecnología, 11.

2 Estudiante de décimo semestre de Derecho de la Universidad de los Andes, y alumna del curso Derecho del Espacio Ultraterrestre. plen misiones, o, aunque hayan cumplido ya su misión, pueden no interrumpir su curso y estropearse en órbita o desintegrarse en el espacio o en las capas densas de la atmósfera (Lachs, 1977 , p. 13). En este sentido, tanto los beneficios como los riesgos que desatan los satélites tienen dimensiones mayores a las de cualquier otra actividad científica (Lachs, 1977, p. 15). De allí la necesidad de promulgar una regulación especial para la actividad espacial.

Debido a esto y a otras razones históricas y políticas que serán explicadas a continuación, las actividades espaciales se han regulado por medio del Derecho Internacional. Al respecto, es de especial importancia la protección de los particulares y de los Estados frente a los daños que se puedan dar en el desarrollo de actividades espaciales. Algunos de estos instrumentos internacionales son la Declaración de los Principios Legales que Rigen las Actividades de los Estados en la Exploración y Utilización del Espacio UItraterrestre, el Tratado del Espacio de 1967 y el Convenio sobre Responsabilidad de 1972. 
En este artículo se analizará el régimen de responsabilidad del Derecho Espacial. Para esto, en primer lugar, se hará referencia a las circunstancias históricas que dieron cabida a esta regulación; en segundo lugar, se expondrán algunos conceptos y elementos jurídicamente relevantes de la misma; en tercer lugar, se esbozará el procedimiento de reclamación por daños causados por objetos espaciales; en cuarto lugar, se hará un enfoque especial de la filosofía que hay detrás de la tipología de responsabilidad en el Derecho Espacial, a partir de un recuento sucinto del desarrollo histórico de la noción de responsabilidad, desde los albores del Derecho Romano, hasta la actualidad. El texto finalizará resaltando la importancia que este régimen y la misma carrera espacial tienen para la humanidad.

\section{ANTECEDENTES HISTÓRICOS Y NORMATIVOS DE LA CARRERA ESPACIAL}

El primer satélite artificial puesto en órbita alrededor de la Tierra fue el Sputnik I, ${ }^{2}$ en 1957 (Lachs, 1977, p. 9). Este hecho marcó el inicio de una era de avances tecnológicos sin precedentes en el ámbito espacial. Sin embargo, la carrera espacial que para ese entonces se consolidaba fue consecuencia de diversos sucesos que marcaron la historia de la humanidad.

Uno de estos sucesos fue la Segunda Guerra Mundial, entre los años de 1939 y 1945 . Du-

2 National Aeronautics and Space Administration - Siddiqi, A. Sputnik I [Fotografía]. Recuperado de http://www.nasa.gov/multimedia/imagegallery/image_feature_924.html rante este conflicto los países dieron prueba del avance tecnológico, por ejemplo, en el campo de la aviación. Además, surgió un temor fundado entre los Estados respecto de la posibilidad de que se desatara una guerra nuclear, con el uso de armas de destrucción masiva que pusieran en peligro la supervivencia de la raza humana.

Este temor dio lugar a que, con el tiempo, los esfuerzos de la comunidad internacional se dirigieran a evitar los conflictos bélicos de la magnitud de las pasadas Guerras Mundiales. Uno de los reflejos del ideal de mantenimiento de la paz y seguridad mundiales fue la reaparición de la Liga de Naciones, que desde la Conferencia de San Francisco de 1945 y hasta la actualidad, se denomina Organización de las Naciones Unidas. Esta organización internacional se consolidaría con el tiempo como la mayor de su clase, y su influencia, en términos normativos, impregnó múltiples áreas del desarrollo humano, incluyendo la carrera espacial.

Sin embargo, la Segunda Guerra Mundial no fue el único acontecimiento histórico que contribuyó a este gran desarrollo tecnológico. La Guerra Fría fue también uno de los factores históricos determinantes para este desarrollo. Este conflicto, prioritariamente ideológico y político, entre la entonces Unión de Repúblicas Socialistas Soviéticas (URSS) y los Estados Unidos (EE. UU.), sentó las bases para que se incursionara en las tecnologías espaciales. En 1957, Ios soviéticos tomaron ventaja en estos adelantos, al ser los primeros en la historia humana en poner en órbita un satélite artificial: el Sputnik I (Kopal, 2008, p. 2). 
En el mismo año, los soviéticos lanzaron también al espacio ultraterrestre el Sputnik II, ${ }^{3}$ el primer vehículo espacial ocupado por un ser vivo, una perra llamada Laika.

Sin embargo, uno de los mayores logros de la URSS se dio el 12 de abril de 1961, fecha en la que por primera vez un ser humano, el soviético Yuri Gagarin, ${ }^{4}$ realizó un viaje al espacio ultraterrestre a bordo del Vostok I.

En adelante, los soviéticos enviaron al espacio cosmonautas $^{5}$ y numerosos objetos espaciales, como el Vostok 2, la estación automática Luna 9, los satélites Soyuz 1, 2, 3, 4 y 5, entre otros.

En vista del gran adelanto obtenido por la URSS en la carrera espacial, el entonces presidente de los Estados Unidos, John F. Kennedy, declaró en 1961 que EE. UU. desarrollaría un programa lunar. El clímax de dicho programa se alcanzó en 1969, cuando los astronautas Neil Armstrong y Edwin Aldrin caminaron por primera vez sobre la Luna. Esta misión, a bordo del Apolo 11 , fue una de las múltiples misiones que consolidaron a EE. UU. como el principal exponente del programa lunar. En adelante, los avances de las potencias en la tecnología espacial no dieron tregua, pues las misiones espaciales se hicieron más amplias, frecuentes e influyentes.

3 NASA. Sputnik 2 [Fotografía]. Recuperado de http://nssdc.gsfc.nasa. gov/nmc/spacecraftDisplay.do?id=1957-002A

4 NASA. Yuri Gagarin: First Man in Space [Fotografía]. Recuperado de http://www.nasa.gov/mission_pages/shuttle/sts1/gagarin_anniversary. html

$5 \quad$ Algunos de ellos fueron: Alexei Leonov, Gherman Titov y Valentina Tereshkova, la primera mujer que viajó al espacio.
Bajo este contexto, la comunidad internacional se vio en la necesidad de fijar unos principios básicos que regularan la actividad espacial de los Estados. Algunos de esos principios son el de cooperación y el de uso pacífico del espacio ultraterrestre, los cuales fueron consignados en las Resoluciones 1348 del 13 de diciembre de 1958 y 1472 del 12 de diciembre de 1959, de la Asamblea General de las Naciones Unidas. Por medio de esta última se creó una comisión de expertos científicos y legales que se encargara de regular la utilización del espacio ultraterrestre. Esta comisión se llamó Comité para la Utilización del Espacio Ultraterrestre. ${ }^{6}$ Hasta el día de hoy, este Comité es el encargado de formular iniciativas legislativas en el ámbito del Derecho Espacial, con base en conocimientos técnicos, científicos y legales.

A nivel normativo, fue relevante la Resolución 1962 del 13 de diciembre de 1963, la cual consignó los principios legales que rigen las actividades de los Estados en la exploración y el uso del espacio ultraterrestre, incluso la Luna y los cuerpos celestes. Pero esta Resolución fue el antecedente del que es considerado el principal instrumento normativo de la actividad espacial. En 1967, la Asamblea General de las Naciones Unidas emitió el Tratado sobre los Principios que Deben Regir las Actividades de los Estados en la Exploración y Utilización del Espacio Ultraterrestre, incluso la Luna y otros Cuerpos Celestes. ${ }^{7} \mathrm{EI}$

6 Copuos, por sus siglas en inglés (United Nations Committee on the Peaceful Uses of Outer Space).

7 Algunos de los principios formulados en el Tratado del Espacio de 1967 son: principio de cooperación: "La exploración y utilización del espacio ultraterrestre, incluso la Luna y otros cuerpos celestes, de- 
contenido de este Tratado se basó en los requerimientos de la carrera espacial para la época.

Entre las motivaciones del Tratado de 1967 tenemos "las grandes perspectivas que se ofrecen a la humanidad como consecuencia de la entrada del hombre en el espacio ultraterrestre", así como "el interés general de toda la humanidad en el proceso de la exploración y utilización del espacio ultraterrestre con fines pacíficos". ${ }^{8}$ Se consideró, por ejemplo, la posibilidad de que se produzcan daños en la Tierra, en el espacio aéreo y en el espacio ultraterrestre.

Teniendo en cuenta esta previsión, los artículos VI y VII del Tratado de 1967 consignaron el principio fundamental de responsabilidad internacional por daños causados por objetos espaciales al que se someten los Estados Partes del Tratado. Según estos artículos, los Estados Partes en el Tratado son responsables internacionalmente de las actividades nacionales que realicen los organismos gubernamentales o entidades no gubernamentales en el espacio ultraterrestre. Además, son responsables por los daños que se ocasionen en la Tierra, en el espacio aéreo o en el espacio ultraterrestre, los Estados desde cuyo territorio o instalaciones se lance un objeto espacial, o que lancen o promuevan el

berán hacerse en provecho y en interés de todos los países, sea cual fuere su grado de desarrollo económico y científico, e incumben a toda la humanidad [...] los Estados facilitarán y fomentarán la cooperación internacional en dichas investigaciones" (artículos I, IX, XX, XXI y XII); principio de no apropiación nacional (artículo II); principio de uso pacífico del espacio ultraterrestre (artículos III y IV); principio por el cual los astronautas se consideran enviados de la humanidad (artículo V). lanzamiento del mismo al espacio ultraterrestre (artículos V y VI).

Estas disposiciones fueron los fundamentos de instrumentos normativos posteriores. El principal, en materia de responsabilidad internacional, es el Convenio sobre la Responsabilidad Internacional por Daños Causados por Objetos Espaciales, aprobado por la Asamblea General de las Naciones Unidas mediante la Resolución 2777 de noviembre 29 de 1971. Posteriormente se analizarán con mayor detenimiento las disposiciones de este estatuto.

En este punto es importante reconocer que, aunque los instrumentos normativos posteriores. El principal en materia de responsabilidad internacional es el Convenio sobre la Responsabilidad, no constituyen una lista excluyente de normatividad. Esto quiere decir que por medio de la Asamblea General de las Naciones Unidas se promulgaron otras regulaciones específicas para aspectos como el registro de objetos espaciales, el salvamento y devolución de astronautas, entre otras. Sin embargo, para efectos de este estudio, solo se hará alusión a las disposiciones relevantes en materia de responsabilidad internacional.

Vistos los antecedentes históricos y normativos que precedieron a la regulación de la responsabilidad internacional en el Derecho Espacial, expondré lo relacionado con la regulación en materia de responsabilidad del Convenio de 1972. 


\section{CONCEPTOS RELEVANTES EN MATERIA DE RESPONSABILIDAD INTERNACIONAL POR DAÑOS CAUSADOS POR OBJETOS ESPACIALES}

El primer concepto relevante a la hora de determinar la responsabilidad por daños causados por objetos espaciales es precisamente el concepto de objeto espacial.

El término objeto espacial se refiere a las partes componentes de un objeto espacial, así como el vehículo propulsor y sus partes (Cocca, 1957, p. 156). Esta definición la encontramos en el artículo I, literal d, del Convenio de Responsabilidad de 1972, reiterado por el artículo I del Convenio sobre el Registro de Objetos Lanzados al Espacio Ultraterrestre, de 1975.

Los objetos espaciales están sometidos a un registro nacional e internacional que nos conduce necesariamente a la noción de "Estado de registro" y "Estado de lanzamiento".

Es Estado de registro el Estado en el cual figura el objeto lanzado al espacio ultraterrestre, el cual retiene su jurisdicción y control sobre tal objeto, así como sobre todo el personal que vaya en él, mientras se encuentre en el espacio ultraterrestre o en un cuerpo celeste. El concepto de Estado de registro es relevante en términos del derecho de propiedad que emana del registro nacional e internacional del objeto espacial (Tratado del Espacio de 1967, artículo VIII; Convenio sobre el Registro de Objetos Lanzados al Espacio Ultraterrestre, artículos II a IV).
Es Estado de lanzamiento todo Estado que lance o promueva el lanzamiento de un objeto espacial, o desde cuyo territorio o instalaciones se lance un objeto espacial (Convenio de Responsabilidad de 1972, artículo I, literal c; Convenio sobre el Registro de Objetos Lanzados al Espacio Ultraterrestre, artículo I).

Surge entonces la cuestión de cuál de los Estados es el llamado a responder por los daños causados por objetos espaciales. El artículo VII del Tratado de 1967 establece que es responsable internacionalmente "todo Estado Parte en el Tratado que lance o promueva el lanzamiento de un objeto al espacio ultraterrestre [...] y todo Estado Parte en el Tratado, desde cuyo territorio o cuyas instalaciones se lance un objeto". Este artículo nos remite al concepto de Estado de lanzamiento que se consigna en el artículo I del Convenio de Responsabilidad, ya mencionado.

Sin embargo, se debe tener en cuenta que gran parte de las actividades espaciales emprendidas actualmente por los Estados involucran a dos o más Estados en la etapa de lanzamiento del objeto. Por ello, el régimen de responsabilidad prevé ciertas circunstancias bajo las cuales la responsabilidad sería conjunta.

Finalmente, como es propio de los elementos constitutivos de la responsabilidad, se debe hacer referencia al concepto de daño que aplica a las actividades espaciales. El artículo I del Convenio de Responsabilidad define el daño como "la pérdida de vidas humanas, las lesiones corporales u otros perjuicios a la salud, así como la pérdida de bienes o los perjuicios causados a 
bienes de Estados o de personas físicas o morales, o de organizaciones internacionales intergubernamentales".

Entonces, para que el daño sea indemnizable, debe ser causado por el objeto espacial o sus componentes en la Tierra, en el espacio aéreo o en el espacio ultraterrestre, incluso la Luna y otros cuerpos celestes (artículo VII, Tratado del espacio, 1967).

\section{RESPONSABILIDAD CONJUNTA EN EL DERECHO ESPACIAL}

Para que se impute responsabilidad a un Estado por daños causados por objetos espaciales, se requiere que el daño se produzca con ocasión del lanzamiento. Esto implica que no solo los lanzamientos exitosos dan lugar a que se atribuya la responsabilidad de los Estados, sino que, también, todo intento de lanzamiento da cabida a que se halle responsable al o los Estado de lanzamiento (Convenio sobre Responsabilidad de 1972, artículo I, literal b).

Ahora bien, un Estado desde cuyo territorio o instalaciones se lanza un objeto espacial, se considerará como participante en un lanzamiento conjunto (artículo V, numeral 3; en adelante, los artículos citados son los del Convenio de Responsabilidad de 1972). El fundamento para que se consolide la responsabilidad conjunta entre los Estados se enuncia en el artículo VI del Tratado de 1967. De acuerdo con este, los Estados son responsables internacionalmente "de las actividades nacionales que realicen en el espacio ultraterrestre, incluso la Luna y otros cuer- pos celestes, los organismos gubernamentales o las entidades no gubernamentales" (cursivas mías) y las organizaciones internacionales.

Cuando se trate de actividades que realiza una organización internacional en el espacio ultraterrestre, incluso la Luna y otros cuerpos celestes, la responsabilidad, de acuerdo con el Tratado, corresponderá a esa organización internacional y a los Estados Partes en el Tratado, que pertenecen a ella.

Las organizaciones internacionales aludidas pueden ser cualquier organización intergubernamental internacional que se dedique a actividades espaciales, que declaren aceptar los derechos y obligaciones previstos en el Convenio de Responsabilidad, y que la mayoría de sus Estados miembros sean Estados Partes del mismo Convenio y del Tratado de 1967 (artículo XXII, numeral 1).

Por otro lado, si el daño fue producido por actividades de organizaciones gubernamentales o no gubernamentales, el responsable es el o los Estados de lanzamiento. Esto se debe a que el Estado de lanzamiento tiene el deber de asegurar que las actividades espaciales se efectúen de conformidad con las disposiciones del Derecho Internacional, al punto de ser autorizadas y fiscalizadas constantemente por el mismo Estado.

En estos dos supuestos de responsabilidad conjunta, los Estados de lanzamiento son solidaria y mancomunadamente responsables de la indemnización a las víctimas (artículo $\mathrm{V}$, numeral 1, y artículo XXII, numeral 3). 
Al existir responsabilidad solidaria, es posible que la víctima del daño demande de cualquiera de los Estado Parte de la organización internacional el pago de la indemnización por los daños causados por el objeto espacial. Además, el Estado al que se haga la reclamación se encuentra en el deber de indemnizar totalmente a la víctima, y, posteriormente, tiene la posibilidad de repetir contra los demás Estados miembros de la organización, si existe acuerdo previo entre ellos al respecto (artículo V, numeral 2).

Se debe tener en cuenta que el procedimiento a seguir si se trata de una organización internacional se lleva a cabo elevando, primero, la reclamación ante la organización internacional, y luego contra los Estados.

La indemnización a la que esté obligado a pagar el Estado de lanzamiento por los daños causados, se determina conforme con el Derecho Internacional, y con los principios de justicia y equidad. El fin de la indemnización es reparatorio, de tal manera que se reponga a la persona, física o moral, al Estado, o a la organización internacional en cuyo nombre se presente la reclamación en la condición que habría existido de no haber ocurrido los daños (artículo XII, numeral 1). De ahí que, a pesar de que ha sido un tema ampliamente discutido, no existe un tope máximo de monto de las indemnizaciones por daños causados por objetos espaciales.

Por último, la indemnización se paga en la moneda del Estado demandante, a menos de que el Estado demandante y el Estado que debe pagar la indemnización acuerden otra forma de indemnización (artículo XIII).
Vistos hasta el momento los elementos de la responsabilidad que aplican al Derecho Espacial, es pertinente hacer alusión a un rasgo especial de esta regulación, y es la tipología de responsabilidad aplicable a los daños causados con ocasión de las actividades espaciales.

\section{TIPOLOGÍA DE RESPONSABILIDAD APLICABLE}

En el Derecho Espacial existe un régimen general de responsabilidad absoluta que aplica a los daños causados por objetos espaciales. Sin embargo, opera bajo ciertas condiciones, que se establecen en el artículo II del Convenio sobre Responsabilidad de 1972, y es que los daños sean causados por un objeto espacial suyo, y que sean causados en la superficie de la Tierra, o a las aeronaves en vuelo (artículo IV, numeral 1, literal a).

Sin embargo, esta tipología de responsabilidad tiene las siguientes excepciones:

- De acuerdo con los artículos III y V, numeral 1, literal $\mathrm{b}$, del mismo Convenio, se establece un régimen de responsabilidad subjetiva, es decir, se debe probar la culpa del Estado cuando el daño se ocasione en el espacio ultraterrestre a otro objeto espacial.

Bajo este supuesto, la carga de la indemnización se reparte entre los Estados solidarios, según el grado de la culpa respectiva. Y si no es posible determinar el grado de la culpa de cada uno de estos Estados, la carga de la indemnización se repartirá por partes iguales entre ellos. 
- Que el Estado de lanzamiento demuestre que los daños son total o parcialmente resultado de negligencia grave, o de un acto de omisión cometido con la intención de causar daños por parte de la víctima (artículo VI).

- Tampoco se aplica a los daños causados a nacionales del Estado de lanzamiento por un objeto espacial de dicho Estado, ni a nacionales de un país extranjero mientras participen en las operaciones de ese objeto espacial, o hayan sido invitados desde el momento de su lanzamiento o en cualquier fase posterior al mismo, hasta su descenso. Tampoco mientras dichos particulares se encuentren en las proximidades inmediatas de la zona prevista para el lanzamiento o la recuperación, como resultado de una invitación de dicho Estado de lanzamiento (artículo VII).

- Finalmente, una excepción a la exoneración de responsabilidad absoluta se encuentra en el artículo VI, numeral 2, del Convenio sobre Responsabilidad. Allí se establece que "no se concederá exención alguna en los casos en que los daños sean resultado de actividades desarrolladas por un Estado de lanzamiento en las que no se respete el derecho internacional". Esto significa que quedaría excluido de ser considerado responsable de manera absoluta el Estado de lanzamiento, en el caso en que el Estado víctima del daño se encontrara en operaciones contra el Derecho Internacional, o los principios del Derecho Espacial.

Estas tipologías de responsabilidad absoluta y subjetiva que establece el Convenio de 1972 son uno de los rasgos diferenciadores más importantes de la responsabilidad en actividades espaciales, por lo que en una sección posterior se hará alusión a la filosofía intrínseca en esta regulación especial. Pero antes de esto, haré alusión al procedimiento de reclamación al que se someten los Estados Parte del Convenio sobre Responsabilidad.

\section{PROCEDIMIENTO DE RECLAMACIÓN}

Los Estados deben agotar la vía diplomática para efectuar las reclamaciones (artículos VIII y IX). En caso de que los Estados involucrados en la controversia no mantengan relaciones diplomáticas, el Estado reclamante puede pedir a otro Estado que presente su reclamación a ese Estado de lanzamiento, o por conducto del Secretario General de las Naciones Unidas, siempre que ambos (el Estado demandante y el Estado de lanzamiento) sean Miembros de las Naciones Unidas (artículo IX).

No es necesario haber agotado los recursos locales de que puedan disponer el Estado demandante o las personas físicas o morales que represente. Tampoco se prohíbe elevar la reclamación ante los tribunales de justicia o ante los tribunales u órganos administrativos del Estado de lanzamiento. La única restricción, relacionada con las reclamaciones al amparo del Convenio de Responsabilidad, es que no se esté tramitando una reclamación ante los tribunales de justicia, o ante los tribunales u órganos administrativos del Estado de lanzamiento, por los mismos daños (artículo XI).

Si no se logra resolver una reclamación mediante negociaciones diplomáticas en un año, a par- 
tir de la fecha en que dé la notificación al Estado de lanzamiento de la documentación relativa a su reclamación, las partes interesadas pueden constituir una Comisión de Reclamaciones (artículo XIV).

Esta Comisión es autónoma en la medida en que ella misma determina su propio procedimiento, el lugar o los lugares en que ha de reunirse, y en la que resolverá todas las demás cuestiones administrativas (artículo XVI, numerales 3 y 4).

Dentro de los dos meses siguientes a la solicitud de constitución de la Comisión de Reclamaciones, se deben nombrar los tres miembros que la compondrán. Uno de ellos es nombrado por el Estado demandante, otro por el o los Estados de lanzamiento, y el tercer miembro, su presidente, debe ser escogido conjuntamente por ambas partes (artículos XV y XVII).

En caso de no lograrse un acuerdo respecto del presidente de la Comisión, los Estados tienen cuatro meses para solicitar al Secretario General de las Naciones Unidas que nombre al presidente en un nuevo plazo de dos meses. $Y$ en caso de que una de las partes no nombre el miembro de la Comisión dentro del plazo fijado, el presidente constituirá por sí solo la Comisión de Reclamaciones, a petición de la otra parte (artículo XVI).

Todos los laudos y decisiones de la Comisión se adoptarán por mayoría de votos (artículo XVI, numeral 5), deben ser motivadas (artículo XIX, numeral 2) y deben versar sobre los fundamentos de la reclamación de indemnización y la cuantía de la indemnización pagadera (artículo XVIII). La decisión tomada por la Comisión es obligatoria para las partes que lo hayan convenido. De no haberse convenido, el laudo definitivo de la Comisión tiene carácter de recomendación, y las partes deben atenderlo de buena fe.

Finalmente, la decisión o laudo es publicado por la Comisión de Reclamaciones, y se expide, entonces, una copia certificada a cada una de las partes, y al Secretario General de las Naciones Unidas (artículo XX).

Existe un término de un año para presentar la reclamación, que cuenta a partir de la fecha en que se produzcan los daños, o en que se haya identificado al Estado de lanzamiento que sea responsable (artículo X). También en el año siguiente a partir de la fecha en que lleguen a su conocimiento los hechos, si el Estado no ha tenido conocimiento de la producción de los daños, o no ha podido identificar al Estado de lanzamiento. Aplica, entonces, un criterio de razonabilidad y diligencia del Estado víctima del daño al momento de establecer si el Estado llegó a tener conocimiento de los hechos, mediante el ejercicio de la debida diligencia (artículo X).

\section{FILOSOFÍA TRAS LA TIPOLOGÍA DE RESPONSABILIDAD APLICABLE AL DERECHO ESPACIAL}

Desde tiempos tempranos, la institución de la responsabilidad ha estado dirigida a la reparación integral de quien ha sido dañado por un hecho. Por ejemplo, lo que hoy asimilamos como 
responsabilidad en la tradición del Derecho Romano, correspondía al respondum (Murillo, 2001, p. 288). Esto implicaba que si un ciudadano incumplía un contrato o cometía un delito, estaba en la obligación de compensar el daño que ocasionase con su conducta (Montoya, 2007, p. 26).

La lex Aquilia, que data del año 287 a. C., reguló en sus capítulos los daños causados por las cosas, el daño causado por el acreedor accesorio al acreedor principal, y las lesiones ocasionadas a los esclavos, animales o a las cosas corporales. Esta ley exigía la intencionalidad de dañar. Estos daños daban lugar a las acciones persecutorias que, en un principio, se asimilan a lo que hoy comprende el Derecho Penal. Sin embargo, fueron un precedente que con el tiempo fue ampliándose a la posibilidad de compensar o resarcir el daño ocasionado por medio del pago de una suma de dinero (Murillo, 2001, p. 290).

La responsabilidad ha sido una institución fundamental que ha estado presente en los regímenes jurídicos que conocemos. Pero lo que para efectos de este estudio es relevante es determinar la justificación de la creación y aplicación de un régimen de responsabilidad especial para el Derecho Espacial.

Podemos ver que, en primer lugar, hay una diferencia fundamental con el concepto genérico de la responsabilidad internacional. De acuerdo con la teoría general, la responsabilidad internacional nace a raíz de un hecho ilícito. ${ }^{9}$ Sin

Comisión General de Reclamaciones. México vs. Estados Unidos. U.N.R.I.A.A. IV. p. 701. Citado en Lachs, M., 1977, p. 165. embargo, es preciso señalar que, en el caso del Derecho del Espacio Ultraterrestre, este presupuesto no es aplicable, puesto que el daño se puede ocasionar en un hecho que no satisfaga la condición de ilicitud (Lachs, 1977, p. 165).

En otras palabras, en el Derecho Espacial no se puede negar la existencia o causación de un daño por el hecho de no haberse omitido un deber o disposición de la ley, o no haberse cometido un delito.

Esta diferencia es plausible y evidente si vemos otros ámbitos del Derecho Internacional, como el Derecho Marítimo y el Derecho Aeronáutico. Bajo estos regímenes, se debía probar la existencia del ilícito, o incluso existían presunciones de culpa que suponían la ocurrencia de un delito. Por ejemplo, en el Convenio de Varsovia de 1929 se dispone que cualquier persona que sufra daños por aviones extranjeros sobre la superficie de la Tierra, tiene derecho a la indemnización, con probar la ocurrencia del daño causado por un vuelo (Convenio para la Unificación de Ciertos Reglamentos Relativos al Transporte Internacional Aéreo, Varsovia, 1929, artículos 17, 18 y 20). Y en el Derecho Marítimo, el artículo VI del Convenio Internacional para la Unificación de Ciertas Normas Jurídicas Relativas a las Colisiones de Bruselas (1910), prescribía el deber de probar el acaecimiento del delito (Lachs, 1977, p. 166).

Surge entonces la pregunta: ¿cuál fue la justificación para crear este régimen especial de responsabilidad? Pues bien, la razón, fundamentalmente, está basada en el grado de peligrosidad 
de la actividad espacial, es decir, el grado de exposición al riesgo que genera (Lachs, 1977, p. 158). Los daños que se pueden causar pueden afectar a toda la humanidad, cambiar el medioambiente de la Tierra, contaminar la atmósfera y producir efectos inconmensurables sobre la vida. Además, hay riesgos propios de los vehículos espaciales, como el riesgo de que colisionen entre ellos o con aviones, que se produzcan interferencias y demás tipos de averías.

Por ello es que esta lex specialis tuvo ciertos antecedentes normativos que reflejaron una filosofía de protección a la víctima del daño altamente garantista. El instrumento especial que hoy conocemos como el Convenio de Responsabilidad, fue antecedido por otros intentos de regulación de responsabilidad presentados por la Subcomisión de Asuntos Jurídicos del Copuos. Sucesivamente y aparentemente sin éxito fueron presentadas múltiples propuestas de países como los Estados Unidos en 1962,10 Bélgica, en 1963, ${ }^{11}$ y Hungría, en $1964 .{ }^{12}$ Pero fueron necesarios diez períodos de sesiones al interior de la Comisión de Asuntos Jurídicos del Copuos para que se llegara a un texto legal que satisficiera la demanda de un régimen especial de responsabilidad para la carrera espacial del momento. El resultado fue la creación del Convenio sobre Responsabilidad Internacional por Daños Causados por Objetos Espaciales, apro-

10 Committee on the Peaceful Uses of Outer Space, UNCOPUOS, Subcomisión de Asuntos Jurídicos (1962). Primer período de sesiones. Informe, pp. 6-8.

11 - (1963). Segundo período de sesiones. Informe, anexo 1, pp. 10-12.

12 - (1964). Tercer período de sesiones. Informe, anexo 2, pp. 7-10. bado por la Asamblea General de las Naciones Unidas, mediante Resolución 2777 (XXVI) del 29 de noviembre de 1971.

En la parte que motiva este Convenio se reconoce expresamente

la necesidad de elaborar normas y procedimientos internacionales eficaces sobre la responsabilidad por daños causados por objetos espaciales y, en particular, de asegurar el pago rápido, con arreglo a lo dispuesto en el presente Convenio, de una indemnización plena y equitativa a las víctimas de tales daños (cursivas mías).

Esto implica que, como lo explica el tratadista Manfred Lachs (1977), se buscaba garantizar los intereses de la víctima, y facilitar la obtención de la indemnización (p. 162). El régimen de responsabilidad absoluta para daños producidos en la Tierra o el espacio aéreo excluye la posibilidad de que el causante del daño justifique su conducta apelando a su diligencia, o a factores como la fuerza mayor o el caso fortuito. Sencillamente, el Estado asume los riesgos que la actividad espacial conlleva (Kerrest, 2005, p. 97).

En la misma parte motivadora del Convenio se deja ver también la pretensión de fortalecimiento de los principios fundamentales del Derecho del Espacio Ultraterrestre, principalmente el de cooperación. El legislador internacional lo expresó así, por estar "convencidos de que el establecimiento de esas normas y procedimientos contribuirá a reforzar la cooperación internacional en el terreno de la exploración y utilización del 
espacio ultraterrestre con fines pacíficos" (cursivas mías).

En suma, la ideología que impregna el régimen de responsabilidad del Derecho Espacial se fundamenta en la protección y garantía de las víctimas de los daños, que son altamente posibles en el marco de las actividades espaciales. El riesgo que la actividad implica per se es significativamente mayor para las potenciales víctimas, de ahí que el derecho sea una herramienta idónea para la protección de dichos intereses de manera amplia y eficiente.

\section{CONCLUSIONES}

El Derecho Espacial se ha visto en la imperante necesidad de regular de manera general la carrera espacial, actividad que, en las últimas siete décadas, ha tenido un desarrollo sobresaliente, y que, entre otros rasgos distintivos, envuelve un grado de peligrosidad superior al habitual para los humanos, el planeta Tierra y los recursos naturales.

Bajo la jurisdicción prácticamente universal de las Naciones Unidas, y de la obligatoriedad de las normas internacionales, se han creado instituciones como el Copuos, y numerosos tratados tendientes a garantizar el desarrollo humano, la integridad de las personas y de los Estados respecto de la actividad espacial.

El principal reflejo de esta filosofía garantista se da en el establecimiento de un régimen de responsabilidad especial para los Estados que par- ticipan en actividades espaciales, pues a diferencia de la regulación tradicional internacional, existe la posibilidad de atribuir a los Estados responsabilidad absoluta por los daños ocasionados por objetos espaciales.

Sin obviar la posibilidad de que esta regulación se modifique, al margen de los avances tecnológicos de las postrimerías de este siglo, dicha regulación es un reflejo de los principios del Derecho Espacial establecidos desde "la Constitución del Espacio", el Tratado de 1967, así como de la cooperación y el uso pacífico del espacio ultraterrestre, recurso natural que ha traído innumerables beneficios a la humanidad.

\section{Referencias}

Cocca, A. A. (1957). Teoría del Derecho Interplanetario. Buenos Aires: Editorial Bibliográfica Argentina.

Committee on the Peaceful Uses of Outer Space, UNCOPUOS, Subcomisión de Asuntos Jurídicos (1962). Primer período de sesiones. Informe, pp. 6-8.

- (1963). Segundo período de sesiones. Informe, anexo 1, pp. 10-12.

- (1964).Tercer período de sesiones. Informe, anexo 2, pp. 7-10.

Convenio para la Unificación de Ciertos Reglamentos Relativos al Transporte Internacional Aéreo. Varsovia, 1929. 
Ferrer, J. (1998). Las consecuencias del hecho ilícito internacional: el proyecto de artículos sobre responsabilidad de los estados aprobado por la C.D.I. en 1996. Alicante: Universidad de Alicante.

Gutiérrez, C. (1979). La responsabilidad internacional por daños en el derecho del espacio. España: Secretaría de Publicaciones - Universidad de Murcia.

Iannini, M.C. (Diciembre de 2012). Los desechos espaciales y su tratamiento en el espacio ultraterrestre. Revista de Derecho Público (29). Bogotá: Universidad de los Andes.

Kerrest, A. (2005). Liability for damage caused by space activities. En M. Benkö y K Schrogl. Space Law: Current legal problems and perspectives for future regulation. The Netherlands: Eleven International Publishing.

Kopal, V. (2008). An Introduction to Space Law. The Netherlands: Wolster Kluwer, Law \& Business.

Lachs, M. (1977). El derecho del espacio ultraterrestre. Madrid: Fondo de Cultura Económica.

Marchán, J. (1987). Derecho internacional del espacio: teoría y política. Quito: Banco Central del Ecuador.

Manrique, A.M. y Morales, V. (Junio de 2012). Responsabilidad internacional por daños causados por objetos espaciales. Revista de Derecho Comunicaciones y Nuevas Tecnolo- gías (7). Bogotá: Universidad de los Andes.

Meloni, G. (1967). International Liability for Space Activity. En Proceedings of the Tenth Colloquium on the Law of Outer Space. Belgrado.

Montoya, F. (2007). Tratado de Derecho Civil. Introducción a la responsabilidad. Bogotá: Universidad Externado de Colombia.

Murillo, A. (2001). La responsabilidad civil. De Roma al Derecho Moderno. Burgos: Universidad de Burgos.

National Aeronautics and Space Administration, NASA. Apollo 11. Recuperado de http://www. nasa.gov/mission_pages/apollo/missions/ apollo11.html

-. Sputnik 1. Recuperado de http://www.nasa. gov/multimedia/imagegallery/image_feature_924.html

-. Sputnik 2. Recuperado de http://nssdc. gsfc.nasa.gov/nmc/spacecraftDisplay. do? id=1957-002A

-. Yuri Gagarin: First Man in Space. Recuperado de http://www.nasa.gov/mission_pages/ shuttle/sts1/gagarin_anniversary.html

Organización de las Naciones Unidas, Asamblea General (Diciembre 13 de 1963). Resolución 1962 (XVII). Declaración de los Principios Jurídicos que Deben Regir las Actividades de los Estados en la Exploración y Utilización del Espacio Ultraterrestre. 
- (Diciembre 19 de 1966). Resolución 2222 (XXI). Tratado Sobre los Principios que Deben Regir las Actividades de los Estados en la Exploración y Utilización del Espacio Ultraterrestre, incluso la Luna y Otros Cuerpos Celestes. Abierto a la firma: enero 27 de 1967; entrada en vigor: octubre 10 de 1967.

- (Noviembre 29 de 1971). Resolución 2777 (XXVI). Convenio sobre la Responsabilidad Internacional por Daños Causados por Objetos Espaciales. Abierto a la firma: marzo 29 de 1972; entrada en vigor: septiembre 11 de 1972.

- (Enero 14 de 1975). Convenio sobre el Registro de Objetos Lanzados al Espacio Ultraterrestre. Resolución 3235 (XXIX).

- (Diciembre 19 de 1967). Acuerdo sobre el Salvamento y la Devolución de Astronautas y la Restitución de Objetos Lanzados al Espacio Ultraterrestre. Resolución 2345 (XXII). 